\title{
Nonlinear Boundary Value Problems for First Order Impulsive Integro-differential Equations
}

\author{
XINZHI LIU \\ Department of Mathematics \\ University of Alberta \\ Edmonton, Alberta, Canada T6G 2G1
}

\begin{abstract}
In this paper, we investigate a class of first order impulsive integrodifferential equations subject to certain nonlinear boundary conditions and prove, with the help of upper and lower solutions, that the problem has a solution lying between the upper and lower solutions. We also develop monotone iterative technique and show the existence of multiple solutions of a class of periodic boundary value problems.
\end{abstract}

Key Words: impulsive integro-differential equation, nonlinear boundary value problem, periodic boundary value problem, monotone iterative technique.

AMS (MOS): 34B15 


\section{Introduction.}

It is well known that the theory of impulsive differential equations is not only richer than the corresponding theory of differential equations but also represent a more natural framework for mathematical modelling of real world phenomena. In spite of this, however, many of its branches are still in an initial stage of their development. This is due, to a large extent, to the difficulties created by the special features possessed by impulsive differential equations such as pulse phenomena, confluence and the loss of autonomy. See $[4,11]$.

One of the ideas in the study of certain higher order boundary value problems for differential equations is to reduce them to boundary value problems for lower order integro-differential equations [1 - 3]. In particular, the second order boundary value problems for differential equations, which has been an object of extensive investigation [ 5 - 7], can be reduced to first order boundary value problems for integro-differential equations. In this paper, we investigate a class of first order impulsive integro-differential equations subject to certain nonlinear boundary conditions and prove, with the help of upper and lower solutions, that the problem has a solution lying between the upper and lower solutions. We also develop monotone iterative technique and show the existence of extremal solutions of a class of periodic boundary value problems for impulsive integro-differential equations. Because of the pecularities mentioned above, the usual tools fail to apply to the problem. Consequently, a very complicated analysis is required.

\section{Preliminaries.}

We consider the impulsive integro-differential equation

$$
\left\{\begin{array}{l}
x^{\prime}=f(t, x, T x), t \neq t_{k}, t \in J \\
\left.\Delta x\right|_{t=t_{k}}=I_{k}(x), k=1,2, \ldots, p
\end{array}\right.
$$


with the following nonlinear boundary condition

$$
q(x(a), x(b))=0
$$

where $J=[a, b], a<t_{1}<t_{2}<\cdots<t_{p}<b, f: J \times R \times R \rightarrow R$ and $I_{k}: R \rightarrow R$ for each $k=1,2, \ldots, p . T$ is a Volterra operator from $P C[J, R]$ into $P C[J, R]$, where $P C[J, R]=\left\{y: J \rightarrow R: y(t)\right.$ is continuous at $t \neq t_{k}, y\left(t^{-}\right)$ and $y\left(t^{+}\right)$exist and $y\left(t^{-}\right)=y(t)$ for $\left.t=t_{k}, k=1,2, \ldots, p\right\}$. We shall assume that $T$ is continuous and monotone nondecreasing and for any bounded set $A \subset$ $P C[J, R], T A$ is bounded. $q: R \times R \rightarrow R$. Let us define the following set of functions $\Omega$ by

$$
\begin{gathered}
\Omega=\left[x: J \rightarrow R: x \text { is continuously differentiable for } t \neq t_{k}, x\left(t^{+}\right),\right. \\
\left.x\left(t^{-}\right) \text {exist and } x\left(t^{-}\right)=x(t) \text { for } t=t_{k}, k=1,2, \ldots, p\right] .
\end{gathered}
$$

A function $\alpha \in \Omega$ is called a lower solution of (2.1) if

$$
\left\{\begin{array}{l}
\alpha^{\prime}(t) \leq f(t, \alpha(t), T \alpha(t)), t \neq t_{k}, t \in J \\
\left.\Delta \alpha\right|_{t=t_{k}} \leq I_{k}\left(\alpha\left(t_{k}\right)\right), \quad k=1,2, \ldots, p
\end{array}\right.
$$

Analogously we can define an upper solution of (2.1) by reversing the inequalities in (2.3). Let us list the following assumptions for convenience.

$\left(A_{0}\right) \quad f: J \times R \times R \rightarrow R$, is continuous for $t \neq t_{k}$,

$$
\lim _{(t, x, y) \underset{c\left(t_{0}, x_{0}, y_{0}\right)}{t<t_{0}}} f(t, x, y)=f\left(t_{0}, x_{0}, y_{0}\right)
$$

and

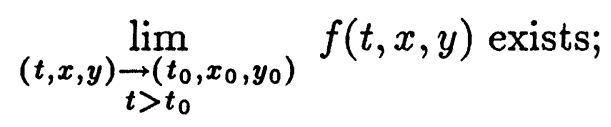

( $\left.A_{1}\right) f(t, x, y)$ is monotone nondecreasing in $y$ for each $(t, x) \in J \times R$ and 
$\sup _{\substack{\alpha(t) \leq x \leq \beta(t) \\ T \alpha(t) \leq y \leq T \beta(t)}}|f(t, x, y)| \leq \lambda(t)$, a.e. on $J$,

where $\alpha(t), \beta(t)$ are lower and upper solutions of (2.1) respectively with $\alpha(t) \leq \beta(t)$ on $J$ and $\lambda \in L^{1}(J)$

$\left(A_{2}\right) \quad I_{k}: R \rightarrow R$ is continuous and nondecreasing in $x$ for each $k=1,2, \ldots, p$;

$\left(A_{3}\right) \quad q: R \times R \rightarrow R$, is continuous and $q(x, y)$ is monotone nonincreasing in $y$ for each $x \in R$.

\section{Method of upper and lower solutions.}

We discuss, in this section, the existence of a solution for the boundary value problem (2.1) - (2.2) by the method of upper and lower solutions.

THEOREM 3.1. Let $\alpha(t), \beta(t)$ be lower and upper solutions of (2.1) and assume that $\left(A_{0}\right)$ to $\left(A_{3}\right)$ hold. Suppose further that

$$
\left\{\begin{array}{l}
q(\alpha(a), \alpha(b)) \leq 0 \\
q(\beta(a), \beta(b) \geq 0
\end{array}\right.
$$

Then the boundary value problem (2.1) - (2.2) possesses a solution $x(t)$ such that $\alpha(t) \leq x(t) \leq \beta(t)$ on $J$

ProOF: Without loss of generality we may assume that $p=1$. We shall first consider the initial value problem

$$
\left\{\begin{array}{l}
x^{\prime}=f(t, x, T x), t \neq t_{1}, t \in J \\
\left.\Delta x\right|_{t=t_{1}}=I_{1}(x) \\
x(a)=x_{a}
\end{array}\right.
$$

and prove that there exists a solution $x(t)=x\left(t, x_{a}\right)$ of $(3.2)$ such that $\alpha(t) \leq$ $x(t) \leq \beta(t)$ whenever $\alpha(a) \leq x_{1} \leq \beta(a)$. For this purpose, we define a function 
$F: J \times R \times R \rightarrow R$ by

$$
F(t, x, T x)= \begin{cases}f(t, \tilde{x}, T \tilde{x})+\frac{x-\alpha(t)}{1+x^{2}}, & \text { if } x<\alpha(t), \\ f(t, \tilde{x}, T \tilde{x}), & \text { if } \alpha(t) \leq x \leq \beta(t), \\ f(t, \tilde{x}, T \tilde{x})+\frac{\beta(t)-x}{1+x^{2}}, & \text { if } x>\beta(t),\end{cases}
$$

where

$$
\tilde{x}= \begin{cases}\alpha(t), & \text { if } x<\alpha(t) \\ x, & \text { if } \alpha(t) \leq x \leq \beta(t) \\ \beta(t), & \text { if } x>\beta(t)\end{cases}
$$

Clearly

$$
\alpha(t) \leq \tilde{x} \leq \beta(t) \text { on } J
$$

and

$$
T \alpha(t) \leq T \tilde{x}(t) \leq T \beta(t) \text { on } J
$$

and

$$
\sup _{(t, x, y) \in J \times R \times I}|F(t, x, y)| \leq \lambda(t), \quad \text { a.e. on } J,
$$

where $I=\{y \in R: T \alpha(t) \leq y \leq T \beta(t)\}$. It is easy to see that the initial value problem

$$
x^{\prime}=F(t, x, T x), x(a)=x_{1}, \text { on } J_{1}=\left[a, t_{1}\right]
$$

has a solution $x(t)$ on $J_{1}$. We claim that $x(t) \leq \beta(t)$ on $J_{1}$. If this is not true, let $m(t)=x(t)-\beta(t)$, then $m(t)$ assumes a positive maximum at some $t_{0} \in\left(a, t_{1}\right]$. Hence there is a $t^{*} \in\left(a, t_{1}\right)$ such that $m\left(t^{*}\right)>0$ and $m^{\prime}\left(t^{*}\right) \geq 0$. Consequently, we have $\tilde{x}\left(t^{*}\right)=\beta\left(t^{*}\right)$ and $T \tilde{x}\left(t^{*}\right) \leq T \beta\left(t^{*}\right)$, which, by the assumptions on $f$, yields

$$
f\left(t^{*}, \tilde{x}\left(t^{*}\right), T \tilde{x}\left(t^{*}\right)\right) \leq f\left(t^{*}, \beta\left(t^{*}\right), T \beta\left(t^{*}\right)\right)
$$


and

$$
\begin{aligned}
m^{\prime}\left(t^{*}\right) & =x\left(t^{*}\right)-\beta\left(t^{*}\right) \leq f\left(t^{*}, \tilde{x}\left(t^{*}\right), T \tilde{x}\left(t^{*}\right)\right)-f\left(t^{*}, \beta\left(t^{*}\right), T \beta\left(t^{*}\right)\right) \\
& +\frac{\beta\left(t^{*}\right)-x\left(t^{*}\right)}{1+x^{2}\left(t^{*}\right)}<0 .
\end{aligned}
$$

This is a contradiction. Similarly, we can prove that $\alpha(t) \leq x(t)$ on $J_{1}$ and therefore it follows that $x(t)$ is a solution of

$$
x^{\prime}=f(t, x, T x), x(a)=x_{a}
$$

on $J_{1}$. Since $\alpha\left(t_{1}\right) \leq x\left(t_{1}\right) \leq \beta\left(t_{1}\right)$ and $I_{1}: R \rightarrow R$ is nondecreasing, we obtain

$$
\alpha\left(t_{1}^{+}\right) \leq x\left(t_{1}\right)+I_{1}\left(x\left(t_{1}\right)\right) \leq \beta\left(t_{1}^{+}\right) .
$$

Setting $y_{1}=x\left(t_{1}\right)+I_{1}\left(x\left(t_{1}\right)\right)$ and repeating the same arguments, we can show that the problem

$$
y^{\prime}=f(t, y, T y), y\left(t_{1}^{+}\right)=y_{1},
$$

has a solution $y(t)=y\left(t, t_{1}, y_{1}\right)$ on $J_{2}=\left[t_{1}, b\right]$ such that $\alpha(t) \leq y(t) \leq \beta(t), t \in$ $J_{2}$. If we extend $x(t)=x\left(t, a, x_{1}\right)$ to $J$ such that

$$
x(t)= \begin{cases}x\left(t, a, x_{a}\right), & \text { if } t \in\left[a, t_{1}\right], \\ y\left(t, t_{1}, y_{1}\right), & \text { if } t \in\left(t_{1}, b\right],\end{cases}
$$

then $x(t)$ is a solution of (3.2) such that

$$
\alpha(t) \leq x(t) \leq \beta(t) \text { on } J
$$

Let $\Omega\left(x_{a}\right)$ be the set of all solutions of (3.2) such that (3.7) holds. Clearly $\Omega\left(x_{a}\right) \neq$ $\phi$. It now remains to show that for some $x \in \Omega$ such that $q(x(a), x(b))=0$. By assumption $\left(A_{3}\right)$ and $(3.1)$, it follows that

$$
q(x(a), x(b)) \leq 0, \quad \text { if } \quad x \in \Omega(\alpha(a)),
$$




$$
q(y(a), y(b)) \geq 0, \quad \text { if } \quad y \in \Omega(\beta(a)) .
$$

We may assume $\alpha(a)<\beta(a)$, for otherwise (3.8) and (3.9) yield $q(x(a), x(b))=0$ which proves the theorem. If the theorem is not true, then we define

$$
M=\{c \in[\alpha(a), \beta(a)]: q(x(a), x(b))<0 \quad \text { for all } x \in \Omega(c)\}
$$

We have $M \neq \phi$ since $\alpha(a) \in M$ so we set $\hat{c}=\sup M$. Let $c_{n} \in M$ with $c_{n} \rightarrow \hat{c}$ and let $x_{n} \in \Omega\left(c_{n}\right)$. Then there is a subsequence which converges piecewise uniformly on $J$ to a solution $x^{0} \in \Omega(\hat{c})$. Since $q\left(x_{n}(a), x_{n}(b)\right)<0$ for all $n$ we have $q\left(x^{0}(a), x^{0}(b)\right) \leq 0$ and therefore $q\left(x^{0}(a), x^{0}(b)\right)<0$. Clearly, $\hat{c}<\beta(a)$ because of relation (3.9). Now let $d_{n}=\hat{c}+\frac{1}{n}<\beta(a)$ for large enough $n$ and let $y_{n} \in \Omega\left(d_{n}\right)$ with $q\left(y_{n}(a), y_{n}(b)\right)>0$ and $x^{0}(t) \leq y_{n}(t) \leq \beta(t)$ on $J$. Then $y_{n}$ has a subsequence which converges piecewise uniformly to a solution $y^{0} \in \Omega(\hat{c})$ and we have $q\left(y^{0}(a), y^{0}(b)\right) \geq 0$. But $y^{0}(a)=x^{0}(a)=\hat{c}$ and $y^{0}(b) \geq x^{0}(b)$ which implies that $q\left(y^{0}(a), y^{0}(b)\right) \leq q\left(x^{0}(a), x^{0}(b)\right)<0$ by the monotoneity condition. This contradiction proves the theorem.

The following two corollaries are easy consequences of Theorem 3.1.

CoROllary 3.2. Let $\alpha(t), \beta(t)$ be lower and upper solutions of (2.1) respectively with $\alpha(t) \leq \beta(a)$ on $J$ and assume that $\left(A_{0}\right)$ to $\left(A_{2}\right)$ hold. Suppose further that

$$
\alpha(a) \leq \alpha(b) \quad \text { and } \beta(a) \geq \beta(b)
$$

Then the periodic boundary value problem (2.1) - (3.11) where

$$
x(a)=x(b)
$$

possesses a solution $x(t)$ such that $\alpha(t) \leq x(t) \leq \beta(t)$ on $J$. 
COROLlaRY 3.3. Let $\alpha(t), \beta(t)$ be lower and upper solutions of (2.1) respectively with $\alpha(t) \leq \beta(t)$ on $J$ and assume that $\left(A_{0}\right)$ to $\left(A_{2}\right)$ hold. Suppose further that

$$
A \alpha(a)-B \alpha(b) \leq r \quad \text { and } A \beta(a)-B \beta(b) \geq r,
$$

where $A^{2}+B^{2} \neq 0, B \geq 0$ and $r$ is a constant. Then the boundary value problem (2.1) - (3.13) where

$$
A x(a)-B x(b)=r
$$

possesses a solution $x(t)$ such that $\alpha(t) \leq x(t) \leq \beta(t)$ on $J$.

\section{Monotone iterative technique.}

For simplicity, we shall consider, in this section, only the periodic boundary value problem $(2.1)-(3.11)$. We shall develop monotone iterative technique which offers a constructive method yielding monotone sequences and investigate the existence of extremal solutions of the PBVP (2.1) - (3.11).

THEOREM 4.1. Let $\alpha(t), \beta(t)$ be lower and upper solutions of (2.1) respectively with $\alpha(t) \leq \beta(t)$ on $J$ and assume that $\left(A_{0}\right)$ to $\left(A_{2}\right)$ hold. Suppose further that

$$
\begin{aligned}
& f(t, x, y)-f(t, \tilde{x}, y) \geq-M(x-\tilde{x}), \quad M>0 \\
& I_{k}(x)-I_{k}(\tilde{x}) \geq-L_{k}(x-\tilde{x}), \quad 0<L_{k} \leq 1, k=1,2, \ldots, p,
\end{aligned}
$$

whenever $t \in J, \alpha(t) \leq \tilde{x} \leq x \leq \beta(t)$ and $[T \alpha](t) \leq y \leq[T \beta](t)$.

Then there exist sequences $\left\{\alpha_{n}\right\},\left\{\beta_{n}\right\}$ which converge monotonically and piecewise uniformly to the minimal and maximal solutions $\rho, \gamma$ of $(2.1)-(3.11)$ on $J$; that is, if $x$ is any solution of $(2.1)-(3.11)$ such that $\alpha(t) \leq x(t) \leq \beta(t)$ on $J$, then

$$
\alpha \leq \alpha_{1} \leq \cdots \leq \alpha_{n} \leq \rho \leq x \leq \gamma \leq \beta_{n} \leq \cdots \leq \beta_{1} \leq \beta \text { on } J \text {. }
$$

Before we proceed to prove Theorem 4.1, it is convenient to prove the following simple lemma. 
LeMmA 4.2. Let $m \in \Omega$ and

$$
\left\{\begin{array}{l}
m^{\prime}(t) \leq-M m(t), t \neq t_{k}, \\
m\left(t_{k}^{+}\right) \leq\left(1-L_{k}\right) m\left(t_{k}\right), k=1,2, \ldots, p, \\
m(a) \leq m(b)
\end{array}\right.
$$

with $M>0$ and $0<L_{k} \leq 1$. Then $m(t) \leq 0$ on $J$.

Proof: Clearly, $m(t) \leq m(a) \prod_{a<t_{k}<t}\left(1-L_{k}\right) e^{-M(t-a)}, t \in J$. To prove the lemma, it is enough to show $m(a) \leq 0$. If this is false, let $m(a)>0$. Then we get

$$
m(a) \leq m(b) \leq m(a) \prod_{k=1}^{p}\left(1-L_{k}\right) e^{-M(b-a)}<m(a),
$$

which is a contradiction.

Proof of Theorem 4.1. For any $\eta$ such that $\eta \in\langle\alpha, \beta\rangle$, where

$$
\langle\alpha, \beta\rangle=[x \in \Omega: \alpha(t) \leq x(t) \leq \beta(t) \text { on } J]
$$

we consider the PBVP for the following linear impulsive differential equation

$$
\left\{\begin{array}{l}
x^{\prime}+M x=\sigma(t), \quad t \neq t_{k}, t \in J \\
\left.\Delta x\right|_{t=t_{k}} \tilde{I}_{k}(x), k=1,2, \ldots, p \\
x(a)=x(b),
\end{array}\right.
$$

where $\sigma(t)=f(t, \eta(t),[T \eta](t))+M \eta(t)$, and $\tilde{I}_{k}(x)=I_{k}\left(\eta\left(t_{k}\right)\right)-L_{k}\left(x-\eta\left(t_{k}\right)\right)$.

It is not difficult to see that the problem (4.1) possesses a unique solution

$$
\begin{aligned}
x(t) & =x(a) \prod_{a<t_{k}<t}\left(1-L_{k}\right) e^{-M(t-a)}+\int_{a}^{t} \prod_{\zeta<t_{k}<t}\left(1-L_{k}\right) e^{M(s-t)}[f(s, \eta(s),[T \eta](s))+M \eta(s)] d s \\
& +\sum_{a<t_{k}<t} \prod_{t_{k}<t_{i}<t}\left(1-L_{i}\right) e^{M\left(t_{k}-t\right)}\left[I_{k}\left(\eta\left(t_{k}\right)\right)-L_{k} \eta\left(t_{k}\right)+\eta\left(t_{k}\right)\right]
\end{aligned}
$$


where

$$
\begin{aligned}
& x(b)=x(a)=\left[1-\prod_{k=1}^{p}\left(1-L_{k}\right) e^{-M(b-a)}\right]^{-1}\left[\int_{a}^{b} \prod_{s<t_{k}<b}\left(1-L_{k}\right) e^{M(s-b)} \times\right. \\
& [f(s, \eta(s),[T \eta](s))+M \eta(s)] d s]+\sum_{k=1}^{p} \prod_{t_{k}<t_{i}<b}\left(1-L_{i}\right) e^{M\left(t_{k}-b\right)}\left[I_{k}\left(\eta\left(t_{k}\right)\right)-L_{k} \eta\left(t_{k}\right)+\eta\left(t_{k}\right)\right] .
\end{aligned}
$$

We define the mapping $A:\langle\alpha, \beta\rangle \rightarrow \Omega$ by $A \eta=x$, where $x(t)$ is the unique solution of (4.1). This mapping will be used to define the desired sequences. Let us first prove that

(i) $\alpha \leq A \alpha, \beta \geq A \beta$,

(ii) $A$ is a monotone mapping on $\langle\alpha, \beta\rangle$.

To prove (i), we set $A \alpha=\alpha_{1}$ and $m=\alpha-\alpha_{1}$. Then we have

$$
m^{\prime}=\alpha^{\prime}-\alpha_{1}^{\prime} \leq f(t, \alpha, T \alpha)-f(t, \alpha, T \alpha)-M\left(\alpha-\alpha_{1}\right)=-M m, t \neq t_{k},
$$$$
m\left(t_{k}^{+}\right) \leq \alpha\left(t_{k}\right)+I_{k}\left(\alpha\left(t_{k}\right)\right)-\alpha_{1}\left(t_{k}\right)-I_{k}\left(\alpha\left(t_{k}\right)\right)+L_{k}\left(\alpha_{1}\left(t_{k}\right)-\alpha\left(t_{k}\right)\right)=\left(1-L_{k}\right) m\left(t_{k}\right),
$$

$$
m(a) \leq m(b)
$$

which implies by Lemma $4.2, m(t) \leq 0$ on $J$, proving $\alpha \leq A \alpha$. Similarly, we can show that $A \beta \leq \beta$. To prove (ii), let $\eta_{1} \leq \eta_{2}, \eta_{1}, \eta_{2} \in\langle\alpha, \beta\rangle$ so that $x_{1}=A \eta_{1}, x_{2}=A \eta_{2}$. Then setting $m=x_{1}-x_{2}$, we get, using $\left(A_{1}\right),\left(A_{4}\right)$ and 
$\left(A_{5}\right)$

$$
\begin{aligned}
m^{\prime} & =x_{1}^{\prime}-x_{2}^{\prime}=f\left(t, \eta_{1}, T \eta_{1}\right)-M\left(x_{1}-\eta_{1}\right)-f\left(t, \eta_{2}, T \eta_{2}\right)+M\left(x_{2}-\eta_{2}\right) \\
& \leq M\left(\eta_{2}-\eta_{1}\right)-M\left(x_{1}-\eta_{1}\right)+M\left(x_{2}-\eta_{2}\right)=-M m, \quad t \neq t_{k}, \\
m\left(t_{k}^{+}\right) & =x_{1}\left(t_{k}^{+}\right)-x_{2}\left(t_{k}^{+}\right)=x_{1}\left(t_{k}\right)+I_{k}\left(\eta_{1}\left(t_{k}\right)\right)-L_{k}\left(x_{1}\left(t_{k}\right)-\eta_{1}\left(t_{k}\right)\right)-x_{2}\left(t_{k}\right) \\
& -I_{k}\left(\eta_{2}\left(t_{k}\right)\right)+L_{k}\left(x_{2}\left(t_{k}\right)-\eta_{2}\left(t_{k}\right)\right) \\
& \leq x_{1}\left(t_{k}\right)-x_{2}\left(t_{k}\right)+L_{k}\left(\eta_{2}\left(t_{k}\right)-\eta_{1}\left(t_{k}\right)\right)-L_{k}\left(x_{1}\left(t_{k}\right)-\eta_{1}\left(t_{k}\right)\right)+L_{k}\left(x_{2}\left(t_{k}\right)-\eta_{2}\left(t_{k}\right)\right) \\
& =\left(1-L_{k}\right) m\left(t_{k}\right), \\
m(a) & =m(b),
\end{aligned}
$$

which yields by Lemma $4.2, m(t) \leq 0$ on $J$, proving (ii).

It is now easy to define the sequences $\left\{\alpha_{n}(t)\right\},\left\{\beta_{n}(t)\right\}$ by $\alpha_{n+1}=A \alpha_{n}, \beta_{n+1}=$ $A \beta_{n}$ with $\alpha_{0}=\alpha, \beta_{0}=\beta$ and to conclude from (i) and (ii) that

$$
\alpha_{1} \leq \alpha_{1} \leq \alpha_{2} \leq \cdots \leq \alpha_{n} \leq \beta_{n} \leq \cdots \leq \beta_{2} \leq \beta_{1} \leq \beta_{0} \text { on } J
$$

It then follows from standard arguments that $\lim _{n \rightarrow \infty} \alpha_{n}(t)=\rho(t), \lim _{n \rightarrow \infty} \beta_{n}(t)=\gamma(t)$ piecewise uniformly on $J$ and that $\rho, \gamma$ are solutions of (2.1) - (3.11) in view of the fact $\alpha_{n}, \beta_{n}$ satisfying (4.1).

To prove that $\rho, \gamma$ are extremal solutions of $(2.1)-(3.11)$, let $x(t)$ be any solutions of $(2.1)-(3.11)$ such that $\alpha(t) \leq x(t) \leq \beta(t)$ on $J$. Suppose that for some $n$, we have $\alpha_{n}(t) \leq x(t) \leq \beta_{n}(t)$ on $J$. Then setting $m(t)=\alpha_{n+1}(t)-x(t)$, 
we obtain

$$
\begin{aligned}
m^{\prime} & =f\left(t, \alpha_{n}, T \alpha_{n}\right)-M\left(\alpha_{n+1}-\alpha_{n}\right)-f(t, x, T x) \\
& \leq M\left(x-\alpha_{n}\right)-M\left(\alpha_{n+1}-\alpha_{n}\right)=-M m, \quad t \neq t_{k}, \\
m\left(t_{k}^{+}\right) & =\alpha_{n+1}\left(t_{k}\right)+I_{k}\left(\alpha_{n}\left(t_{k}\right)\right)-L_{k}\left(\alpha_{n+1}\left(t_{k}\right)-\alpha_{n}\left(t_{k}\right)\right)-x\left(t_{k}\right)-I_{k}\left(x\left(t_{k}\right)\right) \\
& \leq \alpha_{n+1}\left(t_{k}\right)-x\left(t_{k}\right)+L_{k}\left(x\left(t_{k}\right)-\alpha_{n}\left(t_{k}\right)\right)-L_{k}\left(\alpha_{n+1}\left(t_{k}\right)-\alpha_{n}\left(t_{k}\right)\right) \\
& =\left(1-L_{k}\right) m\left(t_{k}\right), \\
m(a) & =m(b) .
\end{aligned}
$$

Then by Lemma 4.2 , we have $m(t) \leq 0$ on $J$ which implies that $\alpha_{n+1}(t) \leq x(t)$ on $J$. Similarly we get $x(t) \leq \beta_{n+1}(t)$ on $J$. Since $\alpha_{0}(t) \leq x(t) \leq \beta_{0}(t)$ on $J$, by induction we conclude that $\alpha_{n}(t) \leq x(t) \leq \beta_{n}(t)$ on $J$ for all $n$. Hence it follows that $\rho(t) \leq x(t) \leq \gamma(t)$ on $J$ by taking limit as $n \rightarrow \infty$ and the proof is complete.

Remark. One of the ideas in the study of certain higher order boundary value problems for differential equation is to reduce them to boundary value problems for lower order integro-differential equations. For example, consider the following second order impulsive differential equation

$$
\begin{cases}u^{\prime \prime}=f\left(t, u^{\prime}, u\right), \quad t \neq t_{k}, & t \in J, \\ \left.\Delta u^{\prime}\right|_{t=t_{k}}=I_{k}\left(u^{\prime}\left(t_{k}\right)\right), & k=1,2, \ldots, p, \\ \left.\Delta u\right|_{t=t_{k}}=L_{k}\left(u\left(t_{k}\right)\right), & k=1,2, \ldots, p,\end{cases}
$$

subject to boundary conditions

$$
u(a)=A, \quad q\left(u^{\prime}(a), u^{\prime}(b)\right)=0
$$




$$
u(b)=B, \quad q\left(u^{\prime}(a), u^{\prime}(b)\right)=0 .
$$

If we set $u^{\prime}=x$ so that $u=T x=A+\int_{a}^{t} x(s) d s+\sum_{a<t_{k}<t} L_{k}\left(u\left(t_{k}\right)\right)$, or

$$
u=T x=\beta+\int_{t}^{b} x(s) d s+\sum_{t<t_{k}<b}\left[g_{k}\left(u\left(t_{k}^{+}\right)\right)-u\left(t_{k}^{+}\right)\right]
$$

where $g_{k}(u)$ is the inverse function of $u+L_{k}(u)$, then the boundary value problems (4.2) - (4.3) and (4.2) - (4.4) reduce to the boundary value problem (2.1) - (2.2). Thus our results obtained in Section 3 and Section 4 can be used to study the second order boundary value problem (4.2) - (4.3) or (4.2) - (4.4). 


\section{References}

1. Aftabizadeh, A.R., Existence and uniqueness theorems for fourth order boundary value problems, J. Math. Anal. Appl. 116 (1986), 415-426.

2. Aftabizadeh, A.R. and Leela, S., Existence results for boundary value problems of integro-differential equations, Proc. Colloquia on Qualitative Theory of Differential Equations at Bolyai Institute, Szeged, Hungary (1984), 23-25.

3. Aftabizadeh, A.R. and Wiener, J., Existence and uniqueness theorems for third order boundary value problems, Rend. Sem. Math. Univ. Padova (1986), 129-141.

4. Bainov, D.D., Lakshmikantham, V. and Simeonov, P.S., Theory of Impulsive Differential Equations, (to appear).

5. Bernfeld, S. and Lakshmikantham, V., "An Introduction to Nonlinear Boundary Value Problems," Academic Press, New York, 1974.

6. Erbe, L.H., Existence of solutions to boundary value problems for second order differential equations, Nonlinear Anal. TMA, 11 (1982), 1155-1162.

7. Erbe, L.H. and Liu, Xinzhi, Existence results for boundary value problems of second order impulsive differential equations, (to appear).

8. Lade, G.S., Lakshmikantham, V. and Vatsala, A.S., "Monotone Iterative Techniques for Nonlinear Differential Equations", Pitman, Belmont, $\mathrm{CH}$ (1985).

9. Lasota, A., On two-point boundary value problems for systems of ordinary nonlinear, first-order differential equations, Ann. Polo. Mat., XXLX (1975).

10. Liu, Xinzhi, Monotone iterative technique for impulsive differential equations in a Banach space, Appl. Math. Phys. Sci. (to appear).

11. Perestyuk, N.A. and Samoilenko, A.M., "Differential Equations with Impulsive Effect," Central Publications, Kiev, U.S.S.R., 1987. 


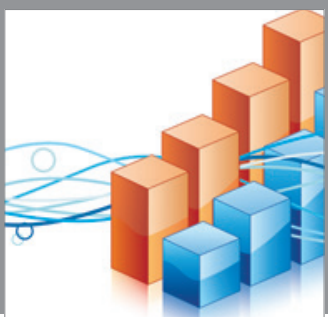

Advances in

Operations Research

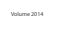

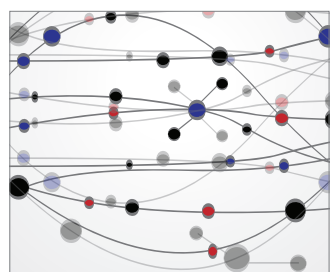

\section{The Scientific} World Journal
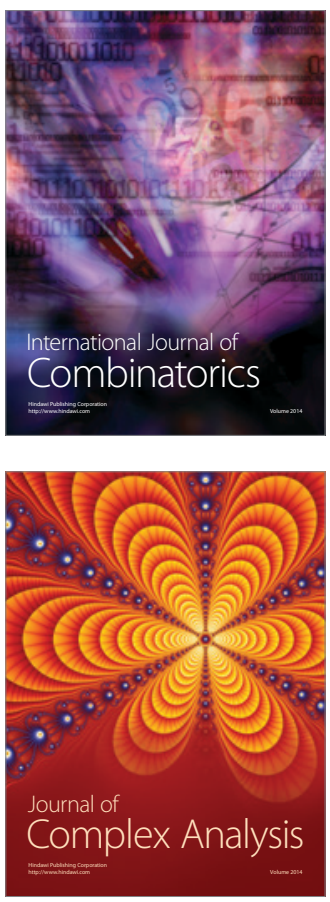

International Journal of

Mathematics and

Mathematical

Sciences
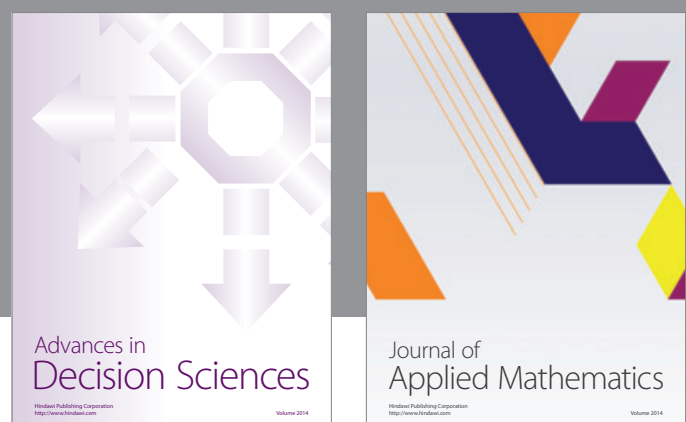

Journal of

Applied Mathematics
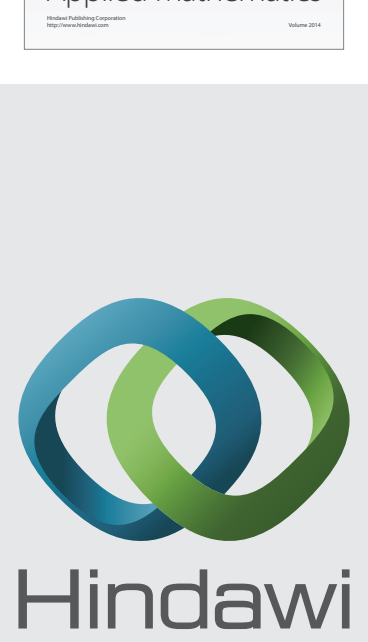

Submit your manuscripts at http://www.hindawi.com
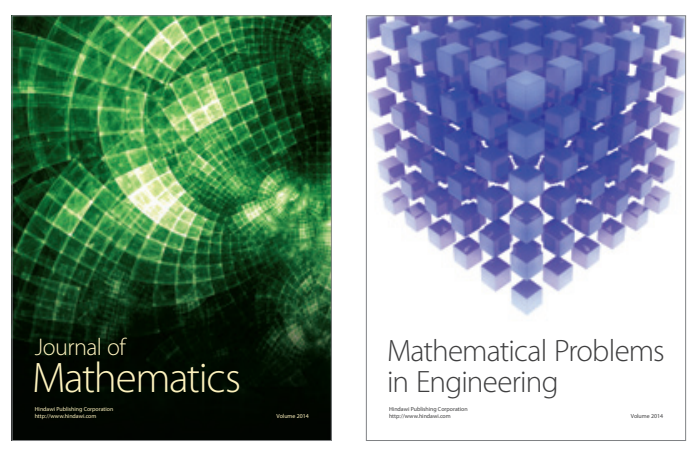

Mathematical Problems in Engineering
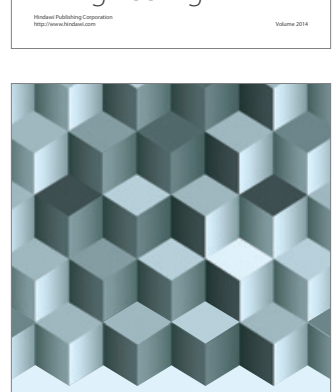

Journal of

Function Spaces
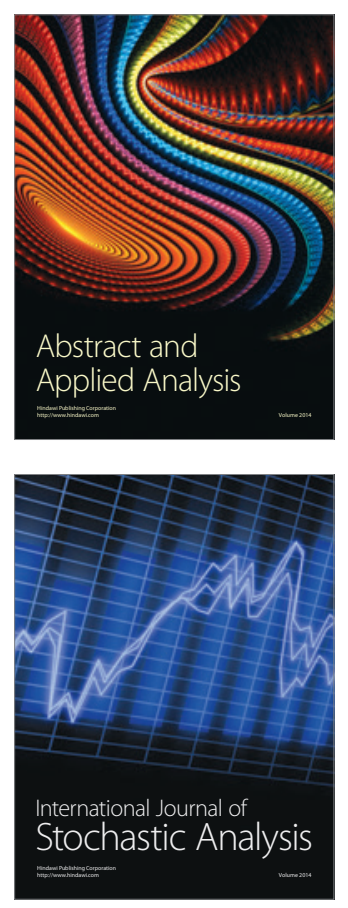

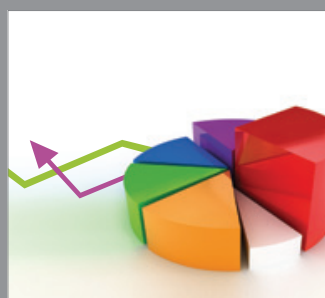

ournal of

Probability and Statistics

Promensencen
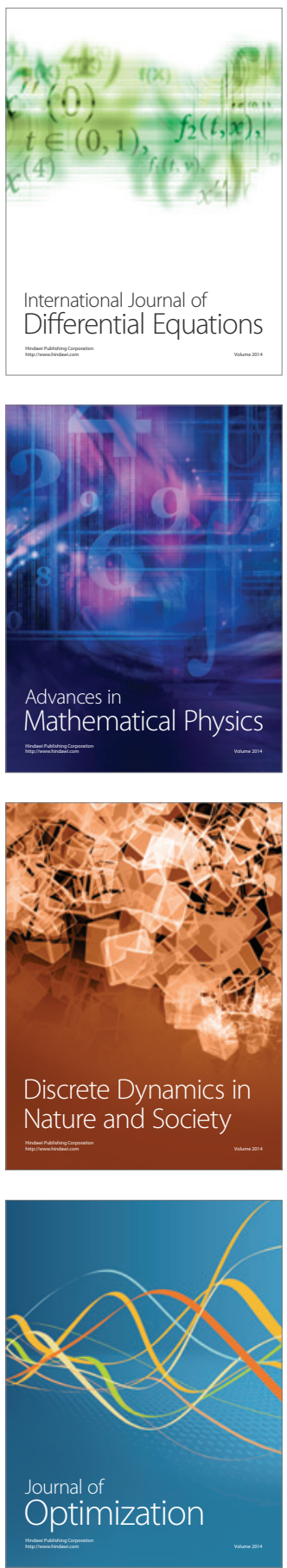